\title{
DIVERSIDAD DE ARAÑAS (ARACHNIDA: ARANEAE) EN EL PARQUE NACIONAL NATURAL ISLA GORGONA, PACÍFICO COLOMBIANO
}

\author{
Alejandro Rico-G. ${ }^{1}$, Juan P. Beltrán A. ${ }^{1}$, Adriana Álvarez D. ${ }^{1}$ \\ \& Eduardo Flórez D. ${ }^{2}$
}

\author{
Biota Neotropica v5 (n1a) - http://www.biotaneotropica.org.br/v5n1a/pt/abstract?inventory+BN007051a2005
}

Recebido em 07/12/2003

Aceito em 01/02/2005

\begin{abstract}
${ }^{1}$ Universidad Nacional de Colombia, Carrera 30 - Calle 45, Bogotá, Colombia. E-mail: aricog@unal.edu.co, jpbeltrana@unal.edu.co, alalvarezd@unal.edu.co

${ }^{2}$ Instituto de Ciencias Naturales, Universidad Nacional de Colombia. A.A. 7495, Bogotá, Colombia. E-mail:

aeflorezd@unal.edu.co
\end{abstract}

\begin{abstract}
In order to establish an approximation to the araneofauna in the Isla Gorgona, located in the Pacific Ocean at approximately $60 \mathrm{~km}$ from the SW coast of Colombia, we took samples in July 2003, using various methods such as capturing the spiders manually, pitfall traps, sweeping with an entomological net, and beating foliage. The samples were taken during the day and night, at four different levels of vertical stratification, in six different habitat which had different states of succession. Sixty-five samples were taken; 1398 spiders were collected and placed in 247 morphospecies, which belong to 34 families. The data were analyzed with 7 species richness estimators: ACE, ICE, Chao 1, Chao 2, first order Jack-knife, second-order Jack-knife, and Bootstrap. The richness estimators varied between each other, with bootstrap having the lowest value (302) and ICE having the highest (504). The failure of the observed species accumulation curve to find an asymptote, showed that more sampling is needed. The most effective method applied was manual collection, with which 33 of the 34 families were collected. The Bray-Curtis similarity index showed that similarity between habitat is very small. Even though Gorgona has a small extension, it presents a high diversity of spiders, which is reflected in the number of families, that is equivalent to $71 \%$ of those found in the whole country.
\end{abstract}

Key words: Diversity, abundance, spiders, Isla Gorgona, inventory, richness indexes.

\section{Resumen}

Para poder establecer una aproximación a la araneofauna de la Isla Gorgona, ubicada en el Océano Pacifico a 60 km de la costa SO de Colombia, tomamos muestras durante el mes de julio del 2003, empleando métodos de captura directa (manual), barridos con red entomológica (sweeping), agitación de follaje (beating) y trampas de caída (pitfall). Los muestreos se realizaron en jornadas diurnas y nocturnas en cuatro niveles de estratificación vertical, los cuales fueron llevados a cabo en seis tipos de habitat contrastantes de acuerdo con su grado de intervención. Fueron tomadas 65 muestras, colectados 1398 individuos agrupados en 247 morfoespecies, pertenecientes a 34 familias. Los datos fueron analizados con 7 estimadores de riqueza: ACE, ICE, Chao 1, Chao 2, Jack-knife de primer orden, Jack-knife de segundo orden y Bootstrap. Los índices de riqueza variaron entre sí, con Bootstrap obteniendo el valor más bajo (302) e ICE obteniendo el más alto (504). La curva de acumulación de especies nunca llegó a una asíntota, demostrando que el muestreo fue insuficiente. El método de captura más efectivo fue la colecta manual, ya que con este método se registraron 33 de las 34 familias encontradas. El índice de Bray-Curtis indicó que la similitud entre los hábitat es muy pequeña. A pesar de comprender un área relativamente pequeña, la Isla Gorgona aloja una significativa diversidad de arañas, lo cual se refleja por que en cuanto a familias se pudo detectar el equivalente al $71 \%$ de las reportadas para el país.

Palabras-clave:Diversidad, abundancia, arañas, Isla Gorgona, inventario, índices de riqueza. 


\section{Introducción}

La diversidad biológica comprende tres atributos: composición, estructura y funcionamiento, los que se expresan en cuatro niveles de organización: paisaje regional, comunidad-ecosistema, población-especies y genético. Cada uno de estos atributos en los diferentes niveles de organización pueden ser caracterizados por indicadores relevantes (Noss 1990). En este trabajo abordamos el atributo de composición a nivel de comunidad-ecosistema en un componente especial de la biota, las arañas (Arachnida: Araneae), caracterizándolo mediante indicadores tales como índices de riqueza específica por ser esta la forma más sencilla de medir la biodiversidad (Moreno 2001).

Las arañas comprenden un grupo faunístico diverso y ampliamente distribuido en todos los ecosistemas terrestres invadiendo incluso algunos ambientes dulceacuícolas. Gracias a que son abundantes en las comunidades y aseguran muestras suficientemente grandes para efectuar análisis numéricos, son modelos apropiados para investigaciones sobre estructura de comunidades, estratificación y sucesión (Turnbull 1973). Son ubicuas, ocupan una gran cantidad de nichos espaciales y temporales, están caracterizadas por una elevada diversidad taxonómica al interior de cada habitat y exhiben respuestas taxón y gremio-específicas a las variaciones ambientales (Toti et al. 2000). Las arañas conforman uno de los grupos más abundantes y diversos en todos los ecosistemas debido a su facilidad para dispersarse y colonizar nuevos habitat (Halaj et al.1998). Su abundancia y diversidad por lo general, están positivamente correlacionadas con la diversidad ambiental a diferentes escalas espaciales (Samu \& Lövei 1995), por lo que se deben encontrar diferencias entre la composición de las comunidades asociadas a diferentes habitat y microhabitat, en un paisaje regional determinado y entre la araneofauna continental y insular.

Las arañas son particularmente diversas en los bosques tropicales, en los cuales se sugiere debe hallarse concentrada alrededor del $80 \%$ de la araneofauna desconocida (Coddington \& Levi 1991). Pese a que las arañas son componentes de gran importancia en el equilibrio ecológico, controlando las poblaciones de invertebrados en estos bosques, el conocimiento que se tiene de ellas se tiene en el neotrópico es aún incipiente. Apenas en las dos últimas décadas se han generado estudios encaminados a obtener información sobre comunidades de arañas en bosques neotropicales: comparándolas con las de bosques templados (Rypstra 1986), en bosques de la amazonía central (Höfer 1990), en el dosel de bosques amazónicos (Höfer et al. 1994) y en la amazonía peruana (Silva 1996, Silva \& Coddington 1996).

En Colombia durante los últimos 30 años se han generado algunos aportes al conocimiento de las arañas del Chocó Biogeográfico, franja continental más próxima al área de estudio; trabajos efectuados por Eberhard (1975, 1977, 1979, 1980) orientados principalmente hacia aspectos de historia natural y comportamiento, y tesis de grado en las universidades locales: Castillo (1981), Bastidas (1992), Villegas (1995) y Vallejo (1997) la mayoría investigaciones ecológicas. Adicionalmente trabajos encaminados a comprender la distribución de las arañas en gradientes altitudinales, verticales y efectos de borde sobre las comunidades de arañas orbitelares (Barriga 1995, Bello 1995, Valderrama 1996) así como estudios sobre estructura y composición de comunidades de arañas hechos en el Departamento del Valle por Flórez (1997, 1998, 1999a, 1999b, 2000).

Este es el primer estudio sistemático sobre araneofauna que se realiza en territorio insular del pacífico colombiano. La presente investigación tiene como objetivo conocer la diversidad de arañas presente en distintos habitat y microhabitat del P.N.N. Isla Gorgona.

\section{Materiales y Métodos}

\section{1 Área de estudio}

El Parque Nacional Natural Isla Gorgona, se localiza entre las coordenadas geográficas $2^{\circ} 47^{\prime}$ y $3^{\circ} 6^{\prime}$ latitud norte; $78^{\circ} 6^{\prime}$ y $78^{\circ} 18^{\prime}$ longitud oeste, comprende una área total terrestre de 1568.4 ha, la mayoría de la isla presenta pendientes pronunciadas y tan solo un $14 \%$ son más o menos planas . Entre la fauna edáfica de Gorgona, los arácnidos presentan estratificación vertical con disminución de sus poblaciones en la profundidad del perfil del suelo, este comportamiento se hace más evidente en las zonas muestreadas en el sector nororiental de la isla. Esta característica hace que la mayor actividad de las arañas se desarrolle al nivel de la superficie del suelo o del horizonte orgánico cuando existe. El horizonte 0 de los suelos de la isla está habitado por un $80 \%$ de insectos y un $20 \%$ de arácnidos (Aguirre \& Rangel 1990). En la isla Gorgona funcionó una penitenciaría hasta 1983, por lo que se encuentran en ella una serie de habitat que presentan distintos grados de intervención humana y por tanto diferentes estados de sucesión.

\section{Mapa de la Isla Gorgona en formato PDF}

Se efectuaron colectas en ambientes contrastantes en la isla de acuerdo con su grado de perturbación:

1) Poblado: Es el más intervenido, comprende un asentamiento humano con perturbación constante. Fueron realizadas colectas en viviendas, vegetación y árboles. 2) Penitenciaría: Está conformado por construcciones abandonadas hace aproximadamente 20 años. Las colectas se llevaron a cabo en los muros y vegetación contigua. 3) 
Playa: Es sometido a modificaciones diarias de extensión por las alta y baja mares. Se muestreó en arena, rocas, y en el ecotono de vegetación limítrofe. 4) Agua Dulce: Este presenta perturbación estacional (aumento de caudales) lo que provoca arrastre de vegetación riparia. Se colectó en vegetación y sobre las rocas. 5) Bosque Secundario: Sectores utilizados en el pasado como cultivos de frutales. Se muestrearon todos los estratos de vegetación incluyendo el dosel. 6) Bosque Primario: Se escogió una zona de bosque prístino en las mayores elevaciones de la isla. Se efectuó el muestreo abarcando todos los estratos, de suelo a dosel.

\subsection{Métodos de los muestreos}

Fueron hechos durante el mes de julio de 2003, con la operación de cuatro colectores simultáneamente, todos presentaban experiencia previa en colección de arañas. Cada muestreo tenía una duración de 2 horas en jornadas diurnas y nocturnas para cada uno de los 6 ambientes expuestos anteriormente. Se efectuó la colección para cada habitat mediante colecta manual en cuatro niveles de estratificación vertical, a saber: 1) Suelo: Incluye hojarasca, piedras, grutas, pasto, arena, troncos y otros. 2) Vegetación Baja: looking down (Coddington et al. 1991), en plantas encontradas entre los 0 y 0,5 metros. 3) Vegetación Alta: looking up (Coddington et al. 1991), en vegetación de 0,5 a 2 metros. 4) Dosel: Con metodología de cuerda simple se muestreó a una altura promedio de 15 metros. Se emplearon en los distintos ambientes barridos con red entomológica de 20 pases dobles no consecutivos en cada muestreo y se utilizó agitación de follaje en 5 árboles en cada ambiente (5 sacudidas o golpes por árbol). Se enterraron a ras del suelo 3 trampas de caída por habitat, con alcohol etílico al 30\%, y permanecieron abiertas durante 48 horas.

Los especímenes fueron determinados a nivel de familia, y para estimativas de riqueza empleamos la metodología propuesta por Oliver \& Beattie (1996b), usamos morfoespecies en cambio de identificar todas las especies por expertos. Para cada morfoespecie consideramos individuos adultos y luego asignamos los juveniles de los cuales aseguramos su correspondencia. Los especimenes fueron ingresados a la Colección Aracnológica del Instituto de Ciencias Naturales, de la Universidad Nacional de Colombia.

\subsection{Manejo de datos}

Para el análisis de los datos usamos el programa EstimateS ${ }^{\circledR}$ versión 7.0 (Colwell 2004), empleamos los siguientes índices de riqueza: ACE, ICE, Chao 1, Chao 2, Jack-knife de primer orden, Jack-knife de segundo orden y Bootstrap. Estos nueve índices no paramétricos estiman el número de especies que faltan por colectar, basándose en la cuantificación de la rareza de las especies colectadas (Toti et al. 2000). ACE (Abundance-based Coverage Estimator) e ICE (Incidence-based CoverageEstimator) son modificaciones de otros estimadores basados en datos de abundancia que superestimaban la riqueza de especies cuando el número de muestras era bajo (Colwell \& Coddington 1994), por lo que están basados en el concepto estadístico de cobertura de muestreo, que se refiere a la suma de las probabilidades de encontrar especies observadas dentro del total de especies presentes, pero no observadas (Colwell 2004). El estimador ACE utiliza para las estimativas diez o menos individuos por muestra, el ICE utiliza especies encontradas en diez o menos muestras (Lee \& Chao 1994). Chao 1 y ACE son estimadores basados en la abundancia, que usan para cuantificar la rareza. Chao 1 utiliza la relación entre el número de Singletones y Doubletones. Por el otro lado ICE, Jack-knife 1, Jack-knife 2, Bootstrap y Chao 2 están basados en incidencia, ellos se basan en presencia y ausencia para cuantificar la rareza. Los estimadores Chao2, Jackknife1, Jackknife2 e Bootstrap, utilizan el número de Uniques y Duplicates (Colwell 2004).Finalmente empleamos análisis de similitud por agrupamientos (clusters) de Bray-Curtis con el programa BioDiversity Pro 2.0 (Mc Aleece 1997), para comparar las composiciones de los habitat y los microhabitat. El índice de Bray-Curtis es una medida de similitud que enfatiza la importancia de las especies que se tienen en común entre los sitios de muestreo (Pileou 1984).

\section{Resultados}

Se encontraron un total de 1398 individuos (469 adultos y 929 juveniles),en 65 muestras incluyendo todos los métodos de captura. Éstos representaron 34 familias (30 Araneomorpha y 4 Mygalomorpha) y 247 morfoespecies. La familia más dominante fue Araneidae (416 individuos) representado un $29,8 \%$ de los individuos colectados, y la familia con mayor número de morfoespecies fue Salticidae (40 mspp.) con un 16,2\% de las morfoespecies colectadas (Tablas I y II). De las familias colectadas, las cuatro más abundantes (considerando aquellas con 100 individuos o más) fueron Araneidae, Tetragnathidae, Salticidae y Theridiidae (Tabla I).

El menor número de morfoespecies se presentó en el habitat playa y la menor abundancia (número de individuos), en agua dulce, mientras que bosque primario presentó los mayores valores para ambos indicadores.

El microhabitat con mayor número de morfoespecies y abundancia fue vegetación alta, mientras que suelo tuvo el menor número de morfoespecies y la menor abundancia, en ambos casos excluyendo dosel y vivienda al no contar con esfuerzos de colecta equiparables (Tabla II).

En los habitat y microhabitat estudiados se presentaron algunas familias con altas abundancias, dadas las condiciones particulares de cada ambiente que favorecen su proliferación. La familia Araneidae fue la más abundante 


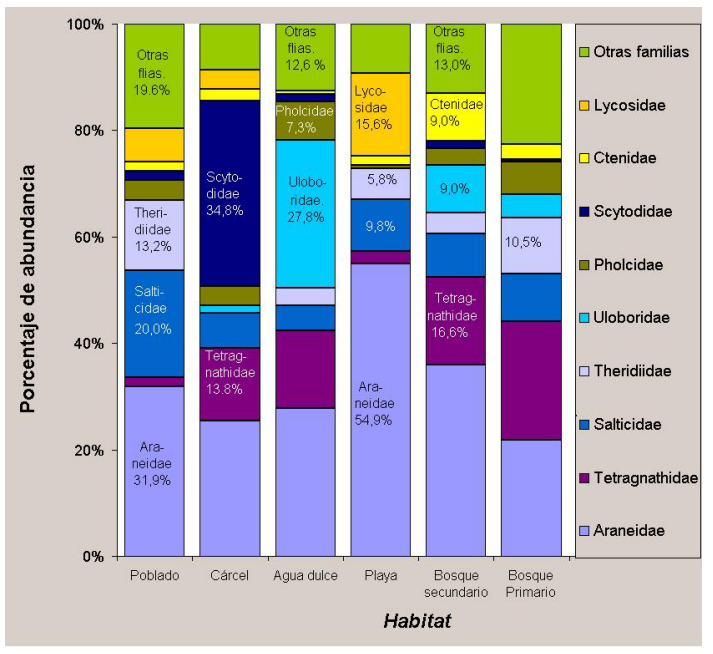

Figura 1. Porcentajes de abundancia para cada familia encontrada en los habitat estudiados. El tamaño de la barra indica el porcentaje de la abundancia de cada familia en particular.

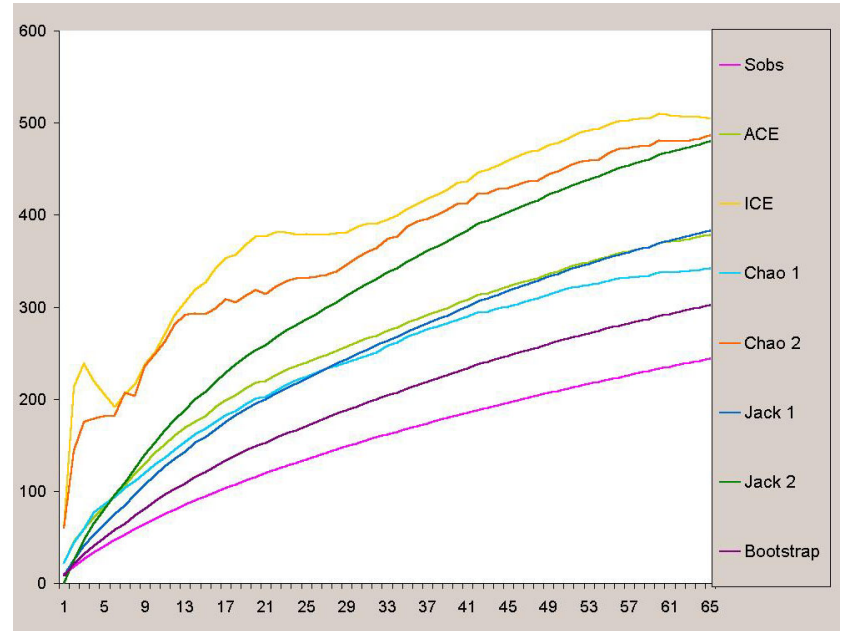

Figura 3. Estimaciones de riqueza de especies de arañas reuniendo las colectas con todos los métodos de captura, en el P.N.N. Isla Gorgona. $S_{o b s}=$ especies observadas.

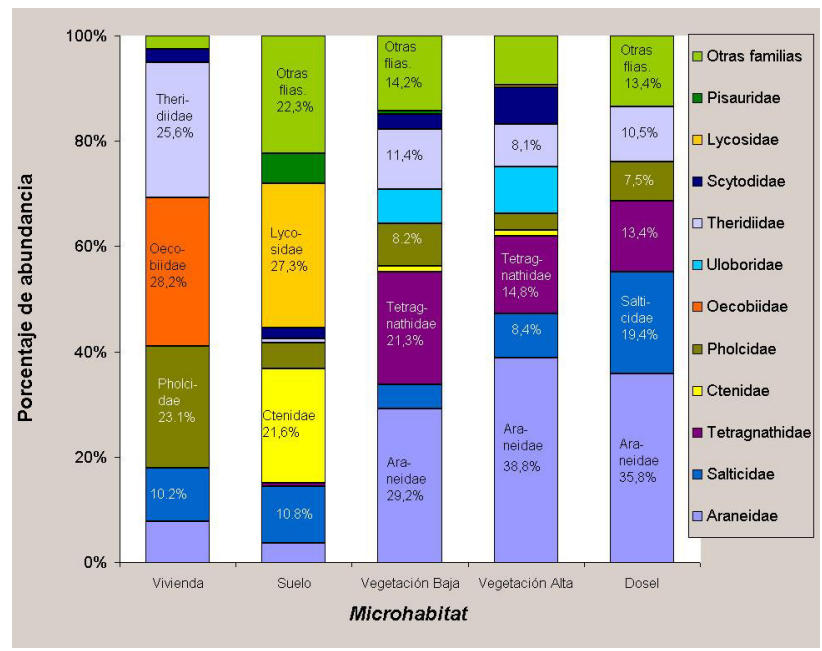

Figura 2. Porcentajes de abundancia para cada familia encontrada en los microhabitat muestreados. El tamaño de la barra indica el porcentaje de la abundancia de cada familia en particular.

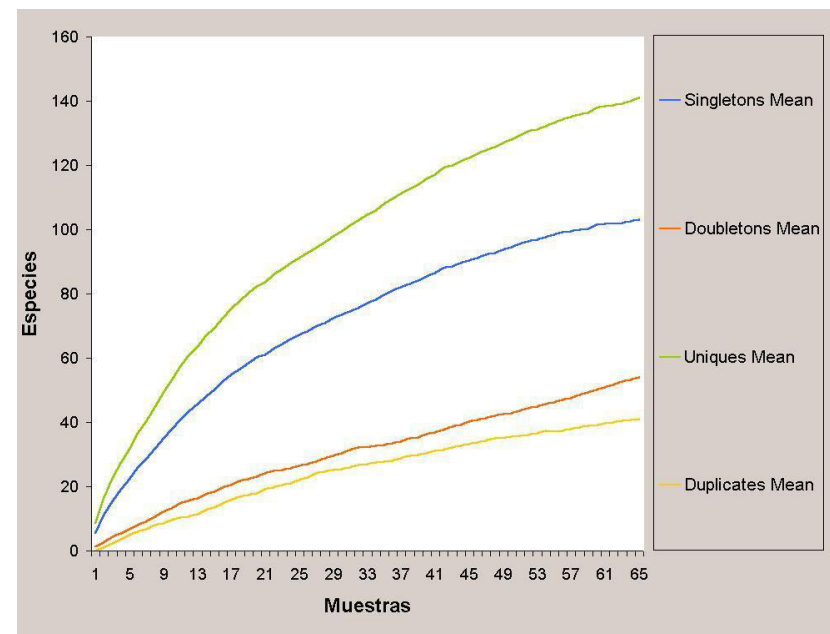

Figura 4. Curvas acumulativas de especies raras de arañas encontradas en el P.N.N. Isla Gorgona. 


\begin{tabular}{|c|c|c|c|c|}
\hline & & \multicolumn{3}{|c|}{ Especímenes } \\
\hline Familia & $\begin{array}{c}\text { No. de } \\
\text { morfoespecies (\%) }\end{array}$ & $\begin{array}{l}\text { Adultos } \\
\text { (n) }\end{array}$ & $\begin{array}{c}\text { Juveniles } \\
\text { (n) }\end{array}$ & Total (\%) \\
\hline Agelenidae & $2(0,9)$ & 8 & 24 & $32(2,3)$ \\
\hline Anapidae & $1(0.5)$ & 1 & 0 & $1(0,07)$ \\
\hline Anyphaenidae & $9(3,6)$ & 2 & 27 & $29(2,1)$ \\
\hline Araneidae & $31(12,6)$ & 89 & 327 & $416(29,8)$ \\
\hline Clubionidae & $2(0,1)$ & 2 & 0 & $2(0,14)$ \\
\hline Corinnidae & $7(2,8)$ & 9 & 2 & $11(0,8)$ \\
\hline Ctenidae & $3(1,3)$ & 9 & 41 & $50(3,6)$ \\
\hline Dipluridae & 2 & 1 & 2 & $3(0,2)$ \\
\hline Gnaphosidae & 1 & 0 & 1 & 1 \\
\hline Hersiliidae & 1 & 1 & 1 & 2 \\
\hline Heteropodidae & $13(5,3)$ & 5 & 17 & $22(1,6)$ \\
\hline Linyphiidae & $22(8,9)$ & 23 & 14 & $37(2,6)$ \\
\hline Liocranidae & 2 & 2 & 0 & 2 \\
\hline Lycosidae & 9 & 13 & 34 & $47(3,4)$ \\
\hline Lyssomanidae & 3 & 3 & 4 & $7(0,5)$ \\
\hline Microstigmatidae & 1 & 0 & 8 & $8(0,6)$ \\
\hline Mimetidae & 1 & 1 & 1 & 2 \\
\hline Miturgidae & 2 & 3 & 3 & $6(0,4)$ \\
\hline Nesticidae & 2 & 3 & 3 & 6 \\
\hline Ochyroceratidae & 1 & 2 & 0 & 2 \\
\hline Oecobiidae & 1 & 8 & 3 & 11 \\
\hline Oonopidae & $5(2)$ & 4 & 2 & 6 \\
\hline Paratropididae & 1 & 0 & 3 & 3 \\
\hline Pholcidae & 7 & 35 & 33 & $68(4,9)$ \\
\hline Pisauridae & $4(1,7)$ & 1 & 10 & 11 \\
\hline Salticidae & $40(16,2)$ & 45 & 95 & $140(10)$ \\
\hline Scytodidae & $10(4)$ & 15 & 47 & $62(4,4)$ \\
\hline Senoculidae & 1 & 1 & 0 & 1 \\
\hline Tetragnathidae & 9 & 57 & 146 & $203(14,5)$ \\
\hline Theraphosidae & 3 & 4 & 0 & $4(0,3)$ \\
\hline Theridiidae & $28(11,4)$ & 47 & 65 & $112(8)$ \\
\hline Theridiosomatidae & 1 & 1 & 2 & 3 \\
\hline Thomisidae & 7 & 4 & 6 & $10(0,7)$ \\
\hline Uloboridae & $15(6,2)$ & 70 & 8 & $78(5,6)$ \\
\hline Totales & $247(100)$ & 469 & 929 & $1398(100)$ \\
\hline
\end{tabular}



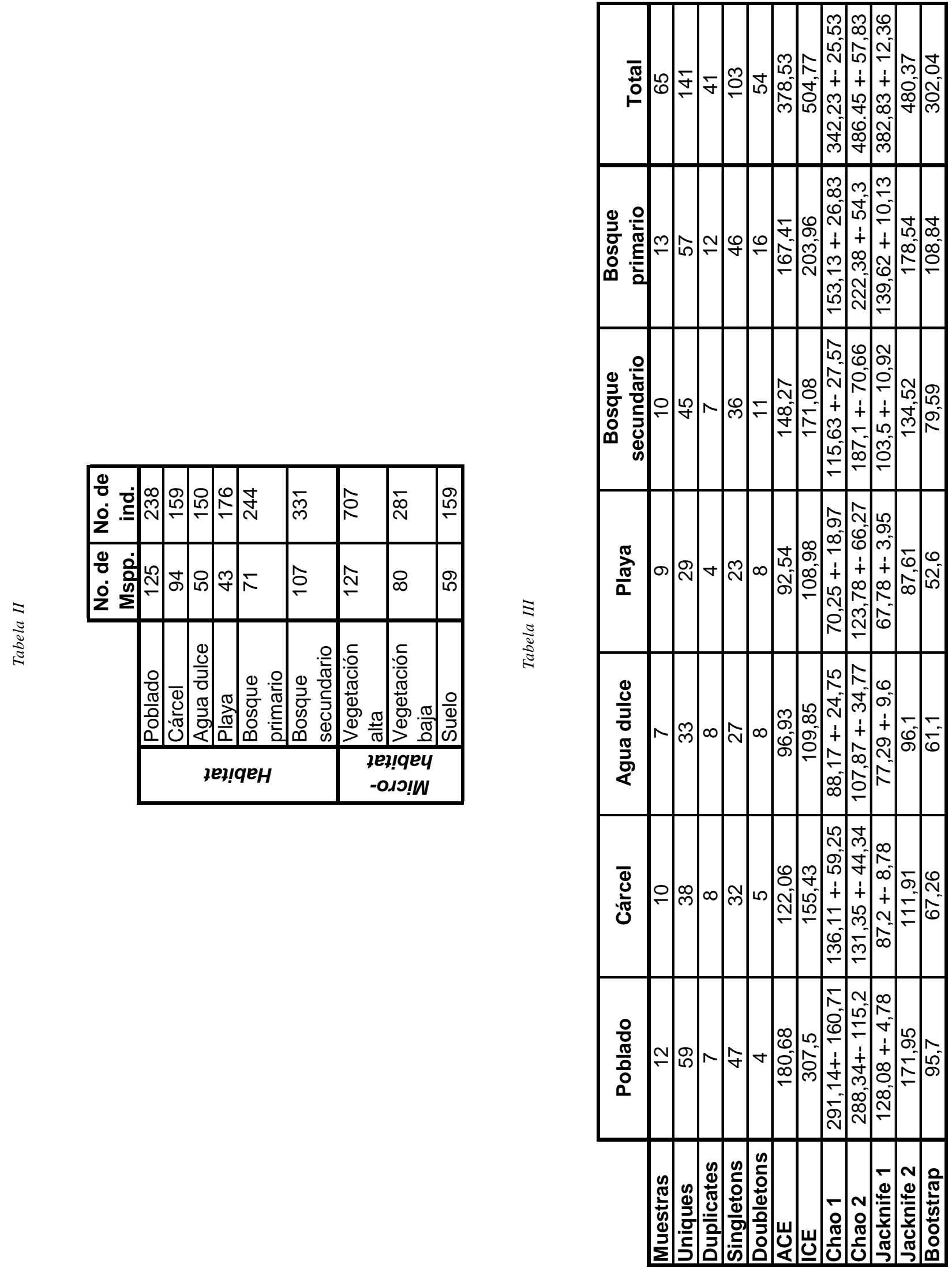

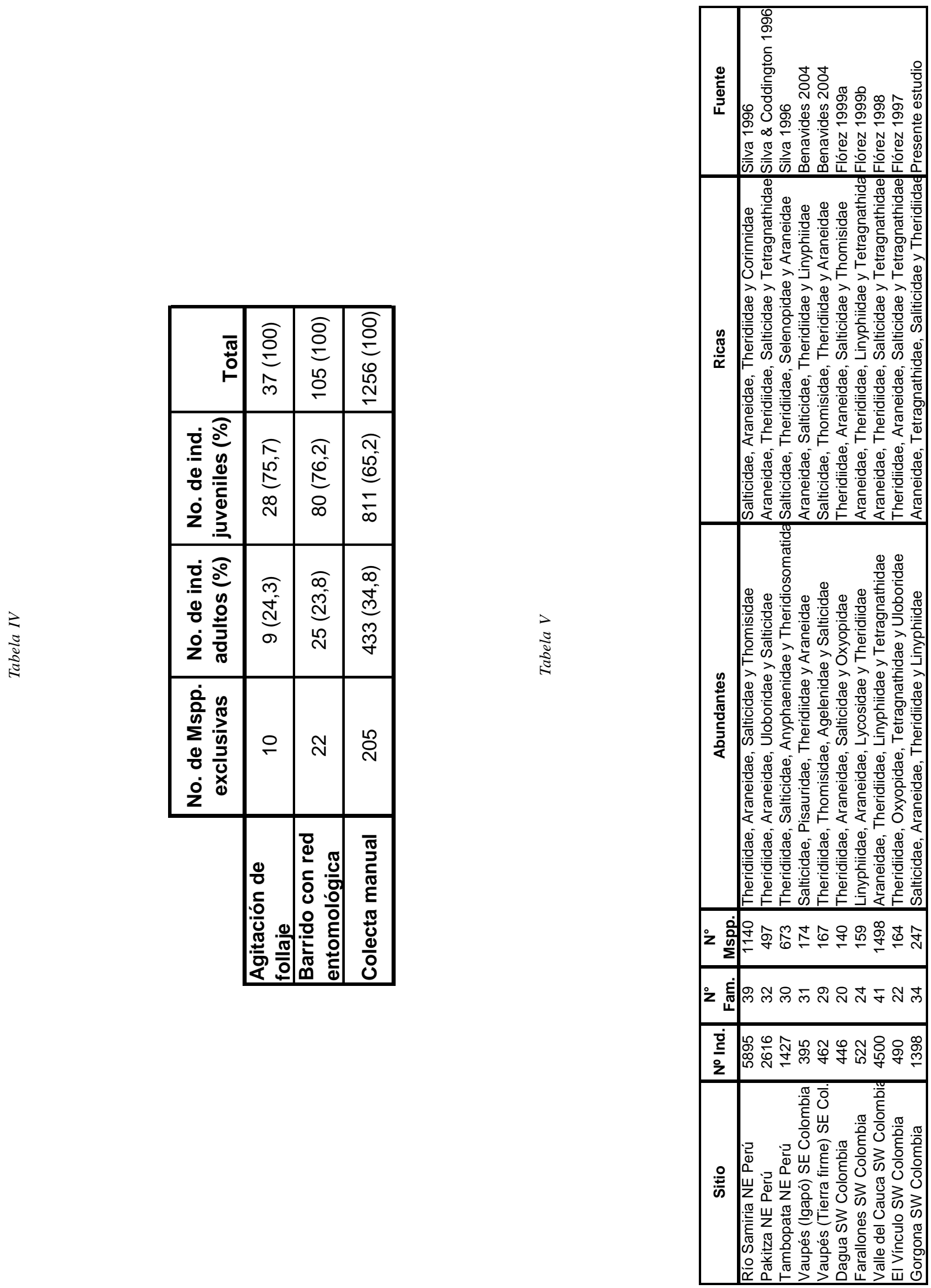

http://www.biotaneotropica.org.br 
en los habitat playa, poblado y bosque secundario, mientras que la familia Tetragnathidae fue la de mayor abundancia en el bosque primario de la isla; esta última familia fue además la que presentó mayor número de individuos en la isla Gorgonilla. En la cárcel se presentó la mayor abundancia de la familia Scytodidae, en agua dulce, la de la familia Uloboridae, y en poblado, la de Oecobiidae (Figura I). En cuanto a los microhabitat, la familia Araneidae fue la de mayor abundancia en la vegetación tanto alta como baja, mientras que en el suelo la presentó Lycosidae. La mayor abundancia en las viviendas humanas la tuvo la familia Oecobiidae, y en dosel, la familia Araneidae (Figura II).

Los estimadores que se presentan en la tabla III se calcularon combinando todos los métodos de captura para cada uno de los habitat. El estimativo más bajo fue Bootstrap con 302 morfoespecies para el parque, y el más alto fue ICE con 504 morfoespecies. Los otros estimativos presentaron variaciones entre sí, Jack-knife 1 y ACE tuvieron valores semejantes, al igual que Chao 2 y Jackknife 2 (Tabla III, figura III). La curva acumulativa de especies $\left(\mathrm{S}_{\mathrm{obs}}\right)$ no alcanza una asíntota, indicando que el número de morfoespecies del parque aumentará con el número de muestras, este mismo comportamiento lo presenta la mayoría de los estimadores utilizados con excepción del ICE y Chao 1 que alcanzan la estabilidad (Figura III).

En cuanto a los estimadores calculados para cada uno de los seis habitat estudiados en la isla, el más bajo siempre fue Bootstrap, con el cual se estimó que el menor número de morfoespecies lo presenta el habitat playa (aproximadamente 53 morfoespecies). El estimador ICE fue el más alto para los habitat poblado, cárcel y agua dulce, mientras que el estimador Chao 2 fue el mayor para playa, bosque secundario y bosque primario, indicando que el bosque primario es el habitat con mayor número de morfoespecies estimadas (aproximadamente 222 mspp.) (Tabla III).

A pesar del número de morfoespecies colectadas, 103 morfoespecies tuvieron un individuo (Singletons) y 54 tuvieron 2 individuos (Doubletons), 141 morfoespecies fueron encontradas en sólo una muestra (Uniques), y 41 morfoespecies en dos muestras (Duplicates). El número Uniques fue mayor que el número de Singletons, Doubletons y Duplicates (Tabla III). En las curvas de morfoespecies raras solamente la de los Singletons tiende a la estabilidad (Figura IV). El habitat con mayor número de Uniques y Singletons fue el poblado, mientras que el bosque primario presentó los valores más altos de $\mathrm{Du}$ plicates y Doubletons (Tabla III).

\subsection{Especimenes}

Al calcular el coeficiente de similitud de Bray-Curtis, ninguno de los habitat fueron similares entre sí (>50\%), el porcentaje de similitud más alto se presentó entre poblado y playa con $34,8 \%$, seguido por los bosques primario y secundario con un valor de $34,4 \%$. Tampoco se presentaron similitudes entre los microhabitat, el mayor porcentaje se obtuvo en vegetación alta y baja con un valor de 34,4\% (resultados no mostrados).

El método de captura más efectivo para el registro de familias fue la colecta manual, con la cual se registraron 33 de las 34 familias encontradas en el parque, tan sólo la familia Gnaphosidae fue reportada con el método de barrido con red entomológica; este último método obtuvo el registro de 16 familias, mientras que los métodos de agitación de follaje y trampas de caída arrojaron 12 y 4 familias respectivamente. El método de captura con el cual se obtuvo el mayor número de individuos y la mayor proporción de adultos fue también la colecta manual, sin embargo con todos los métodos se obtuvo mayor proporción de juveniles que de adultos (Tabla IV). La mayor cantidad de especies exclusivas la presentó de nuevo el método de colecta manual, sin embargo los otros métodos lograron a su vez una cantidad elevada de especies detectadas tan sólo por éstos (Tabla IV).

\section{Discusión}

Este estudio estableció los primeros datos de diversidad de familias de arañas presentes en distintos habitat y microhabitat del PNN Isla Gorgona. La isla aloja una considerable diversidad de arañas, lo cual se advierte porque en cuanto a familias detectamos el equivalente al $71 \%$ de las reportadas para el país.

Debido a que los trabajos efectuados en islas del pacífico se han centrado en grupos muy restringidos de arañas, y tan sólo en islas oceánicas remotas (e.g. Gillespie 2002), discutimos nuestro estudio con investigaciones desarrolladas en bosques continentales de características similares. Los datos obtenidos en el presente estudio muestran resultados similares a los presentados en los trabajos de Silva (1996) y Silva y Coddington (1996) para Perú, y las revisiones efectuadas por Nentwig (1993) para Panamá, y por Flórez \& Sánchez (1995) para Colombia.

Las familias con mayor número de morfoespecies en el parque fueron Salticidae, Araneidae y Theridiidae; comparando con otros estudios en el neotrópico, vemos que estas tres familias son también las de mayor riqueza en otras localidades (Tabla V). Las familias con mayor número de individuos en este estudio fueron Araneidae, Tetragnathidae, Saliticidae y Theridiidae; por lo general en otros estudios, las familias Araneidae, Salticidae y Theridiidae presentan alta abundancia, sin embargo la familia Tetragnathidae sólo es abundante en los estudios del suroccidente colombiano (Flórez 1997, 1998) (Tabla V).

Para Colombia los inventarios de especies son incipientes y principalmente basados en la literatura (GiledeMoncayo \& Bello-Silva 2000) por esta razón empleamos unidades taxonómicas reconocibles (morfoespecies) ya que 
han demostrado ser útiles para una rápida estimación de la biodiversidad (Oliver \& Beattie 1993). Oliver \& Beattie (1996b) llevaron a cabo un análisis comparativo de métodos para medir la diversidad, usaron especies identificadas por expertos y morfoespecies identificadas por inexpertos. Sus resultados fueron similares en términos de evaluación de biodiversidad basada en especies o morfoespecies, lo que significa que una aproximación precisa a las morfoespecies hace posible la evaluación de la biodiversidad de un ecosistema. Más aún, en especial se ha encontrado que las estimaciones de la riqueza de hormigas y arañas varia muy poco entre los inventarios de morfoespecies y de especies (Oliver \& Beattie 1996a). Mediante el uso de morfoespecies, nuestra investigación mostró viable la aplicación de estimadores de riqueza, rareza y abundancia para evaluar la diversidad en la isla.

Los estimadores basados en el concepto estadístico de cobertura de la muestra son relativamente nuevos, y se encuentran en un período de experimentación (Longino et al. 2002). En la mayoría de los casos estos estimadores varían en su tendencia a la estabilidad, sólo ICE y Chao 1 alcanzan su asíntota, siendo el Chao 1 el que la logra con mayor rapidez. Al utilizar estos estimadores, es importante tener en cuenta que su naturaleza no ha sido plenamente probada (Colwell \& Coddington 1994). La curva del estimador ICE muestra un acelerado crecimiento inicial, seguido de una caída, sólo tiende a alcanzar la estabilidad con altos números de muestras; esto indica que este estimador es susceptible al número de muestras empleado y que por tanto no es confiable con un bajo número de muestras.

Un buen estimador debe cumplir las siguientes características: 1) alcanzar la estabilidad (o aproximarse) con menos muestras de las que se requieren para que la curva acumulativa de especies se estabilice, 2) su estimación no debe diferir ampliamente de la de los otros estimadores, 3) su estimación debe ser cercana a una extrapolación visual razonable de la asíntota de la curva de acumulación de especies (Toti et al. 2000). De acuerdo con estas características, nuestro mejor estimador fue Chao 1 ya que se aproximó más a la estabilidad. El estimador Bootstrap difirió de los otros estimadores, por calcular el menor número de especies para la isla, también presentó el menor valor para cada uno de los habitat estudiados; el pobre desempeño de este estimador también ha sido reportado en otros estudios (Colwell \& Coddington 1994, Toti et al. 2000, Bragagnolo \& Pinto-da-Rocha 2003).

Las especies raras juegan un papel importante en la generación de la mayoría de los estimadores al garantizar la confiabilidad de los resultados obtenidos, dado que los estimadores de riqueza están basados en su cuantificación. La mayoría de las curvas acumulativas de especies raras para todo el parque no declinan ni se estabilizan con el aumento del número de muestras, únicamente la curva de Singletons tiende a la estabilidad pero no declina. Este hecho está directamente relacionado con que la mayoría de las curvas de los estimadores, y la curva acumulativa de especies, no alcancen la asíntota (Toti et al. 2000). Dado que la mayoría de las curvas de los estimadores de riqueza y de especies raras no alcanzan una estabilidad, se requiere un mayor esfuerzo de muestreo para que los estimadores den una aproximación más significativa de la riqueza de especies en el parque.

Ya que las arañas son sensibles a pequeños cambios en la estructura de su habitat, esperábamos que los ambientes con un estado más avanzado de sucesión como los bosques primario y secundario fueran contundentemente los medios más diversos, sin embargo el poblado y el bosque primario fueron los ambientes con el mayor número de individuos, de morfoespecies y de especies raras, pese a que el poblado es el habitat con mayor grado de intervención y el bosque primario es el más conservado, resultado similar al obtenido por Whitmore et al. (2002) en Sudáfrica.

Se afirma que la estructura física de un ambiente influye en la selección del mismo por una araña, postulado demostrado al probar que existe una relación directa entre la complejidad estructural de un habitat y su diversidad de especies (Uetz 1979). En el poblado se pudo observar que la estructura física de los lugares que habitan las arañas rara vez es alterada, entonces probablemente el poblado y el bosque primario fueron los ambientes más diversos porque son los que ofrecen mayor heterogeneidad espacial por ende mayor disponibilidad de nichos, indicando que el poblado, a pesar de su alto grado de intervención, es un ambiente en la isla con alta complejidad estructural. El tipo de habitat parece influir en la composición de arañas a nivel de familia, dado que familias del mismo gremio buscan estar en ambientes similares, no obstante, a nivel de especies el tipo de ambiente no parece influir en la composición de la comunidad (Whitmore et al. 2002). En nuestro estudio, cada habitat presentó familias y morfoespecies únicas, lo que indica que para conservar la diversidad de arañas en la isla todos ellos son importantes.

Las elevadas tasas de captura con el método de colecta manual en comparación con los otros métodos son congruentes con lo reportado en estudios anteriores en el país, al igual que los reducidos números de individuos colectados con trampas de caída (v.g. Flórez 1998, Flórez 1999a). Esto último puede estar relacionado con la elevada tasa de precipitación en la isla (Aguirre \& Rangel 1990), con la escasa capa de hojarasca que caracteriza a los bosques tropicales (Bultman y Uetz 1982), así como con las bajas cantidades de materia orgánica de sus suelos, que inciden en la densidad de microfauna del suelo y la hojarasca (Seastedt 1984).

Pese a que se haya considerado las densidades poblacionales de faunas tropicales como estables, gracias a ser comunidades complejas y diversas, y a la relativa constancia de las condiciones climáticas, estudios 
fenológicos de la artropofauna tropical han demostrado que existen cambios regulados por las variaciones interanuales de los períodos de lluvia/sequía (Levings \& Windsor 1985, Pearson \& Derr 1986). Por esto los presentes resultados deben ser complementados con futuros estudios en la isla para establecer una aproximación más cercana a la riqueza real de su araneofauna. Sugerimos intensificar los nuestros muestreos en dosel por ser este habitat donde pueden encontrarse especies que no se presentan en los estratos bajos del bosque y más aún aquellas que permanecen desconocidas para la ciencia (Erwin 1983, Basett 2001).

Es interesante resaltar que pese a comprender un área relativamente pequeña, la Isla Gorgona aloja un sorpresivamente alto porcentaje de las familias reportadas para el país, y que tal como lo evidencian los estimadores de riqueza y la curva acumulativa de especies faltan muchas más por registrar. Se recomienda efectuar estudios que utilicen otros métodos de colecta, en especial aquellos que examinen la hojarasca, e intensificar los empleados por nosotros. De igual forma clasificar las arañas encontradas en gremios, con el fin de obtener análisis más detallados sobre la organización de las comunidades asociadas a los diferentes ambientes, teniendo en cuenta sus variaciones en estados sucesionales y su complejidad estructural, que como evidenciamos con nuestro trabajo alojan familias y morfoespecies únicas o por lo menos que presentan dominancias (de individuos o morfoespecies) características en cada habitat, razón por la cual son todos importantes para conservar la diversidad de arañas en el P.N.N. Isla Gorgona.

\section{Agradecimentos}

A nuestro compañero de campo Hannier Pulido, por su invaluable ayuda. Al Ministerio del Medio Ambiente, Unidad Administrativa de Parques Nacionales Naturales, por su apoyo logístico, permisos de trabajo y colecta en la isla. Al Instituto de Ciencias Naturales, Universidad Nacional de Colombia, por el préstamo de los implementos de trabajo tanto de campo como de laboratorio. A Edwin Barrios y Alejandra Rodríguez, por su ayuda en el manejo y presentación de los resultados. Y a los trabajadores, pobladores e investigadores del Parque Nacional Natural Isla Gorgona por todo su respaldo y colaboración.

\section{Referencias Bibliográficas}

AGUIRRE, J. C. \& RANGEL, O.C. (Eds.). 1990. Biota y ecosistemas de Gorgona. Editorial Fondo FEN Colombia.

BARRIGA, B.J. 1995. Cambios en la diversidad de arañas constructoras de telas orbiculares (Araneae: Orbicularie) a lo largo de un gradiente altitudinal, en el Parque Nacional de Munchique, Cauca. Trabajo de grado. Pontificia Universidad Javeriana, Bogotá.
BASSET, Y., CHARLES, E., JAMMOND, D.S. \& BROWN, V.K. 2001. Short-term effect the canopy openness on insect herbivores in a rainforest in Guyana. J. Appl. Ecol. 38: 1045-1058.

BASTIDAS, H. 1992. Aracnofauna en el Valle del Cauca, en algodonero y arroz: reconocimiento, incidencia, consumo y efecto de insecticidas. Tesis de grado. Universidad Nacional de Colombia, Palmira.

BELLO, S.J.C. 1995. Efectos de borde sobre la distribución de arañas orbitelares (Araneae: Orbicularie) en un bosque de niebla de la Reserva Natural La Planada, Nariño. Trabajo de grado. Pontificia Universidad Javeriana, Bogotá.

BENAVIDES, L.R. 2004. Arañas (Aranae) de dosel en dos bosques del Vaupés amazonia colombiana. Trabajo de grado. Universidad Nacional de Colombia, Bogotá.

BRAGAGNOLO C. \& PINTO-DA-ROCHA, R. 2003. Diversidade de opiliões do Parque Nacional da Serra dos Órgãos, Rio de Janeiro, Brasil (Arachnida: Opiliones). Biota Neotropica 3(1): http://www.biotaneotropica.org.br/v3n1/pt/ abstract?article+BN00203012003

BULTMAN, T.L. \& UETZ, G.W. 1982. Abundance and community structure of forest floor spiders following litter manipulation. Oecologia 55: 34-41.

CASTILLO, J.A 1981. El mimetismo de las telarañas artificiales. Tesis de grado. Universidad del Valle, Cali.

CODDINGTON, J.A., GRISWOLD, C.E., SILVA, D., PEÑARANDA, E., \& LARCHER, S. 1991. Designing and testing sampling protocols to estimate biodiversity in tropical ecosystems. pp. 44-60. In: The Unity of Evolutionary Biology (E.C. DUDLEY, Ed). Dioscorides Press.

CODDINGTON, J.A \& LEVI, H.W. 1991. Systematics and evolution of spiders. Ann. Rev. Ecol. Syst. 22: 565-592.

COLWELL, R.K. \& J.A. CODDINGTON. 1994. Estimating terrestrial biodiversity through extrapolation. Phil. Trans. Royal Soc. 345: 101-118.

COLWELL, R.K. 2004. EstimateS. Statistical Estimation of Species Richness and Shared Species from Samples. Version 7. User's Guide and application at: http// :viceroy.eed.uconn.edu/estimates

ERWIN, T.L. 1983. Tropical forest canopies, the last biotic frontier. Bull. Entomol. Soc. Amer. 29: 14-19.

EBERHARD, W.G. 1975. The "inverted ladder" orb web of Scoloderus sp. and the intermediate orb of Eustala (?) sp., Araneae: Araneidae. J. Nat. Hist. 9: 93-106.

EBERHARD, W.G. 1977. “Rectangular orb” webs of Synotaxus (Araneae: Theridiidae). J. Nat. Hist. 11: 501-507. 
EBERHARD, W.G. 1979. Rates of egg production by tropical spiders in the field. Biotropica 11(4): 292-300.

EBERHARD, W.G. 1980. The natural history and behavior of the bolas spider Mastophora diizzydeani, (Araneidae). Psyche 87: 143-153.

FLÓREZ, D.E. \& SANCHEZ, H. 1995. La diversidad de los arácnidos en Colombia, aproximación inicial. In: O. Rangel (ed), Colombia, Diverdidad Biótica, I. Instituto de Ciencias Naturales, Universidad Nacional, Inderena, FES, FEN. Santafé de Bogotá.

FLÓREZ, D.E. 1997. Estudio de la comunidad de arañas del bosque seco tropical de la estación biológica “El Vínculo”. Cespedesia 22(69): 37-57.

FLÓREZ, D.E. 1998. Estructura de comunidades de arañas (Araneae) en el departamento del Valle, sur occidente de Colombia. Caldasia 20(2): 173-192.

FLÓREZ, D.E. 1999a. Estructura y composición de una comunidad de arañas (Araneae) en un bosque muy seco tropical de Colombia. Bol. Entomol. Venez. 14(1): 37-51.

FLÓREZ, D.E. 1999b. Estudio de comunidades de arañas (Aranae) del Parque Nacional Farallones de Cali, Colombia. Cespedesia, 23(73-74): 99-113.

FLOREZ, D.E. 2000. Comunidades de arañas de la región pacífica del departamento del Valle del Cauca, Colombia. Revista Colombiana de Entomología 26(3-4): 77-81.

GILEDE-MONCAYO, O. \& BELLO-SILVA, J.C. 2000. La familia Araneidae (Araneoidea: Orbicularie) en el departamento del Meta, Colombia. Biota Colombiana 1(1): $125-130$.

GILLESPIE, R.G. 2002. Biogeography of spiders on remote oceanic islands of the Pacific: archipelagoes as stepping stones?. Journal of Biogeography 29: 655-662.

HALAJ, J., ROSS, D.W. \& MOLDENKE, A.R. 1998. Habitat structure and prey availability as predictors of the abundance and community organization of spiders in western Oregon forest canopies. J. Arachnol. 26: 203-220.

HÖFER, H. 1990. The spider community of a Central Amazonian blackwater inundation forest (Igapó). Acta Zool. Fenn. 190: 173-179.

HÖFER, H., BRESCOVIT, A.D., ADIS, J. \& PAARMAN, W. 1994. The spider fauna of Neotropical tree canopies in Central Amazonia. First results. Studies on Neotropical Fauna and Environment 29: 23-32.

LEE, S.M. \& CHAO, A. 1994. Estimating population size via sample coverage for closed capture-recapture models. Biometrics (50): 88-97.

LEVINGS, S.C. \&WINDSOR, D.M. 1985. Litter arthropod populations in a tropical deciduous forest: relationships between years and arthropod groups. J. Anim. Ecol. 54: 61-69
LONGINO, J.T., CODDINGTON, J. \& COLWELL, R.K. 2002. The ant fauna of a tropical rain forest: estimating species richness tree different ways. Ecology 83(3): 689-702.

MC ALEECE, N. 1997. BioDiversity Professional Beta 2.0. The Natural History Museum.

MORENO, C.E. 2001. Métodos para medir la biodiversidad. M\&T-Manuales y Tesis SEA, vol. 1. Zaragoza.

NENTWIG, W. 1993. Spiders of Panama. Fauna \& Flora Handbook. The Sandhill Crane Press.

NOSS, R.F. 1990. Indicators for monitoring biodiversity: a hierarchical approach. Cons. Biol. 4: 355-364.

OLIVER, I. \& BEATTIE, A. J. 1993. A Possible Method for the Rapid Assessment of Biodiversity. Cons. Biol. 7(3): 562-568.

OLIVER, I. \& BEATTIE, A. J. 1996a. Invertebrate Morphospecies as Surrogates for Species: A Case Study. Cons. Biol. 10(1): 99-109.

OLIVER, I. \& BEATTIE, A. J. 1996b. Designing a cost-effective invertebrate survey: A test of methods for rapid assessment of biodiversity. Ecol. Appl. 6(2): 594-607.

PEARSON, D.L. \& DERR, J.A. 1986. Seasonal patterns of lowland forest floor arthropod abundance in Southeastern Peru. Biotropica 18: 244-256.

PILEOU, E.C. 1984. The interpretation of ecological data. Wiley, New York.

RYPSTRA, A.L. 1986. Web spiders in temperate and tropical rainforests: relative abundance and environmental correlates. Am. Midl. Nat. 115 (1): 42-51.

SAMU, F. \& LÖVEI, G.L. 1995. Species richness of a spider community: extrapolation from simulated increasing sampling effort. European J. Entomol. 92: 633-638.

SEASTEDT, T.R. 1984. The role of microarthropods in descomposition and mineralization processes. Ann. Rev. Entomol. 29: 25-46.

SILVA, D. 1996. Species composition and community structure of Peruvian rainforest spiders: a case study from a seasonally inundated forest along the Samiria river. Rev Suisse Zool., vol. hors série: 597-610.

SILVA, D. \& CODDINGTON, J.A. 1996. Spiders of Pakitza (Madre de Dios, Perú): Species Richness and Notes on Community Structure. Pp. 253-311. In. D. E. Wilson and A. Sandoval (Eds.). Manu: the Biodiversity of Southeastern Peru. Smithsonian Institution.

TOTI, D.S., COYLE, F.A\& MILLER, J.A. 2000. A structured inventory of appalachian grass bald and heath bald spider assemblages and a test of species richness estimator performance. J. Arachnol. 28: 329-345. 
TURNBULL, A.L. 1973. Ecology of the true spiders. Ann. Rev. Entomol. 18: 305-348.

UETZ, G.W. 1979. The influence of variation in litter habitats on spider communities. Oecologia 40: 29-42.

VALDERRAMA, C.A. 1996. Comparación de la distribución vertical de arañas constructoras de telas orbiculares en tres zonas de un bosque nublado. Tesis de MSc en Ecología. Universidad de los Andes, Bogotá.

VALLEJO, M.I. 1997. Estructura de una comunidad de arañas orbitelares (Araneae) en sistemas agroforestales del Bajo Anchicayá, Pacífico colombiano. Tesis de grado. Universidad del Valle, Cali.

VILLEGAS, M.E. 1995. Determinación de los hábitos alimenticios de las arañas del género Leucauge (Tetragnathidae) en el Departamento del Valle del Cauca. Tesis de grado. Universidad del Valle, Cali.

WHiTMORE, C., SLOTOW, R., CROUCH, T.E. \& DIPPENAAR-SCHOEMAN, A.S. 2002. Diversity of spiders (Aranae) in a savanna, Northern province, South Africa. J. Arachnol. 30: 344- 356.

Título: Diversidad de arañas (Arachnida: Araneae) en el Parque Nacional Natural Isla Gorgona, Pacífico Colombiano

Autores: Alejandro Rico G., Juan P. Beltrán A., Adriana Álvarez D.\& Eduardo Flórez D.

Biota Neotropica, Vol. 5 ( número 1a): 2005

http://www.biotaneotropica.org.br/v5n1a/pt/ abstract?inventory+BN007051a2005

Recebido em 07/12/2003 - Aceito em 01/02/2005

ISSN 1676-0603

http://www.biotaneotropica.org.br 